\title{
Synthesis of Optimal Algorithms of Recognition of Group Air Targets in Airborne Radar Systems
}

\author{
Alexandr V. Bogdanov ${ }^{\text {a }}$, Victor N. Bondarev, \\ Oleg V. Vasiliev ${ }^{a}$, Evgeniy N. Garin ${ }^{b}$, \\ Denis V. Zakomoldin ${ }^{a}$, Sergey S. Korotkov ${ }^{a}$, \\ Igor V. Lyutikov*b and Vladimir N. Lyaporov ${ }^{\mathrm{a}}$ \\ ${ }^{a}$ VKO Military Academy named Marshal \\ of the Soviet Union Georgiy Zhukov \\ 50 Zhigareva Str., Tver, 170100, Russia \\ ${ }^{b}$ Siberian Federal University \\ 79 Svobodny, Krasnoyarsk, 660041, Russia
}

In the article by Bayesian optimality criteria algorithms for the recognition stage, the secondary signal processing and numerical model of group air targets, the nature of its flight in radar systems, airborne, built by pulse-Doppler (ID) principle of signal processing of a long time coherent accumulation. In this case, it is assumed that there is information about the state of group air targets, the resulting signal processing in radar at the stage of primary processing of radar (radar) signals reflected from the group of air targets.

Keywords: pulse-Doppler radar, group aerial target, recognition

Citation: Bogdanov A.V., Bondarev V.N., Vasiliev O.V., Garin E.N., Zakomoldin D.V., Korotkov S.S., Lyutikov I.V., Lyaporov V.N. Synthesis of optimal algorithms of recognition of group air targets in airborne radar systems, J. Sib. Fed. Univ. Eng. technol., 2017, 10(2), 154-168. DOI: 10.17516/1999-494X-2017-10-2-154-168.

(C) Siberian Federal University. All rights reserved

* Corresponding author E-mail address: lyutikovigor@mail.ru 


\title{
Синтез оптимальных алгоритмов
}

\section{распознавания групповых воздушных целей \\ в радиолокационных системах воздушного базирования}

\author{
А.В. Богданов ${ }^{\text {a }}$, В.Н. Бондарев ${ }^{\text {, }}$ \\ О.В. Васильев ${ }^{a}$, Е.Н. Гарин ${ }^{\sigma}$, Д.В. Закомолдин ${ }^{a}$, \\ С.С. Коротков ${ }^{\text {a }}$ И.В. Лютиков ${ }^{\sigma}$, В.Н. Ляпоров ${ }^{a}$ \\ ${ }^{a}$ Военная академия ВКО \\ имени маршала Советского Союза Г.К. Жукова \\ Россия, 170100, Тверь, ул. Жигарева, 50 \\ ${ }^{6}$ Сибирский федеральный университет \\ Россия, 660041, Красноярск, пр. Свободный, 79
}

\begin{abstract}
В статье при байесовском критерии оптимальности синтезированы алгоритмы распознавания на этапе вторичной обработки сигналов численного и типового состава групповой воздушной цели (ГВЦ), характера её полёта в радиолокационных системах (РЛС) воздушного базирования, построенных по импульсно-доплеровскому (ИД) принципу обработки сигналов с длительнымм временем их когерентного накопления. При этом предполагается, что имеется информация о состоянии ГВЦ, полученная в результате обработки сигналов в РЛС на этапе первичной обработки радиолокационных (РЛ) сигналов, отражённых от ГВЦ.
\end{abstract}

Ключевые слова: импульсно-доплеровская РЛС, групповая воздушная цель, распознавание.

\section{Введение}

Одним из путей расширения информационных возможностей ИД РЛС с длительным временем когерентного накопления отраженных сигналов, позволяющим осуществить разрешение по доплеровской частоте элементов ГВЦ, находящихся в главном луче диаграммы направленности РЛС, является распознавание численного и типового состава самолётов в группе из класса «самолёты с турбореактивными двигателями (ТРД)», а также характер их полёта по принципу «стационарный полёт-манёвр в группе-манёвр составом группы». Основным информативным параметром отражённого от ГВЦ радиолокационного сигнала в ИД РЛС на этапе его вторичной обработки при распознавании численного состава ГВЦ и характера её полёта является изменяющаяся во времени доплеровская частота, обусловленная отражениями сигнала от планера каждого самолёта группы, а при распознавании типового состава ГВЦ - дополнительно изменяющаяся во времени доплеровская частота, обусловленная отражениями РЛ-сигнала от вращающихся лопаток первой ступени компрессора низкого давления (КНД) при РЛ-наблюдении ГВЦ в переднюю полусферу или турбины - при РЛ-наблюдении в заднюю полусферу. При этом предполагается, что предварительное распознавание численного и типового состава ГВЦ осуществлено на этапе первичной обработки сигналов в ИД РЛС воздушного базирования. Поэтому на этапе вторичной обработки РЛсигналов в ИД РЛС уточняется информация о состоянии ГВЦ, полученная на этапе первичной обработки сигналов.

В статье понимается: 
под оценкой численного состава ГВЦ - количество разрешаемых и сопровождаемых траекторий доплеровских частот (ТДЧ), обусловленных скоростями сближения носителя РЛС и каждого самолёта из состава их группы;

под распознаванием характера полёта ГВЦ - оценка набора параметров полёта ГВЦ по принципу «стационарный полёт-манёвр в группе-манёвр составом группы»;

под распознаванием типового состава ГВЦ - разнос (разность) по доплеровской частоте ТДЧ, обусловленных отражениями РЛ-сигнала от планеров самолётов из класса «самолёты с турбореактивными двигателями» и вращающихся лопаток первой ступени КНД (турбины) их силовых установок.

Данный разнос по доплеровской частоте для каждого типа двигателя, установленного на самолёте, постоянен, что позволяет определить тип двигателя, установленного на каждом самолёте группы, а по нему - тип самого самолёта в группе. Таким образом, на основе одного наблюдения на выходе системы первичной обработки РЛ-сигналов с длительным временем когерентного накопления необходимо принять несколько решений о состоянии ГВЦ (её численном и типовом составе, характере полёта элементов ГВЦ). Общие подходы к синтезу подобных алгоритмов приведены, например, в [1, 2].

Цель работы - синтезировать оптимальные алгоритмы распознавания состояния ГВЦ в РЛС воздушного базирования на этапе вторичной обработки РЛ-сигналов при предварительном распознавании состояния ГВЦ на этапе первичной обработки РЛ-сигналов.

\section{1. Синтез оптимального алгоритма распознавания численного состава групповой воздушной цели и характера её полёта}

\section{1. Постановка задачи на синтез алгоритма}

Пусть в каждый дискретный момент времени на выходе первичной обработки РЛ-сигналов на основе их узкополосной доплеровской фильтрации (длительного времени когерентного накопления) возможно появление $m$ сигналов $\left(\mathrm{m}=\hat{\mathrm{m}}^{\text {(по) }}-1, \hat{\mathrm{m}}^{\text {(по) }}, \hat{\mathrm{m}}^{(\text {по) }}+1\right.$, где $\hat{\mathrm{m}}^{(\text {по) }}-$ количество ВЦ в группе, оцененное на этапе первичной обработки (индекс «по»), соответствующих $m$ значениям доплеровских частот $F_{i}(i=\overline{1, m})$. Примем гипотезу $\Gamma_{m j}$ и поставим ей в соответствие ситуацию о том, что имеет место $m$ разрешаемых доплеровских частот $F_{m j}$, соответствующих в общем случае $j$-му $(j=\overline{1, L})$ характеру (набору параметров) полёта воздушных целей. При этом справедливо следующее условие:

$$
P\left(\Gamma_{m j}\right)=P_{m j}, \quad \sum_{j=1}^{L} \sum_{\hat{m}^{(n o)}-1}^{\hat{m}^{(n o)}+1} P_{m j}=1,
$$

где $P_{m j}-$ вероятность справедливости гипотезы $\Gamma_{m j}$.

В этом случае оцениваемый вектор состояния будет составным и иметь в непрерывном времени следующий вид

$$
\hat{\gamma}_{m j}=|\hat{m}, \hat{j}, \hat{T}(t)|^{\mathrm{T}}
$$

где $\hat{\gamma}_{m j} \in G, G$ - дискретно-непрерывное множество составного вектора состояния; $\hat{T}(t)$ обобщённый вектор оценённых значений частных векторов состояния 


$$
\begin{aligned}
\hat{T}(t)=\mid \hat{T}_{\hat{m}^{(n o)}-11}(t), \ldots, \hat{T}_{\hat{m}^{(n o)}-1 L}(t) ; \hat{T}_{\hat{m}^{(n o)} 1}(t), \ldots, \hat{T}_{m j}(t), \ldots \\
\\
\ldots, \hat{T}_{\hat{m}^{(n o)} L}(t) ; \hat{T}_{\hat{m}^{(n o)}+11}(t), \ldots, \hat{T}_{\hat{m}^{(n o)}+1 L}(t) \mid
\end{aligned}
$$

где

$$
\hat{T}_{m j}(t)=\left|\hat{F}_{1 j}(t), \hat{F}_{2 j}(t), \ldots, \hat{F}_{i j}(t), \ldots, \hat{F}_{m j}(t)\right|^{\mathrm{T}} .
$$

В дискретном представлении наблюдаемый сигнал на выходе первичной обработки сигналов на $k$-м дискрете при гипотезе $\Gamma_{m j}$ будет иметь следующий вид:

$$
\Gamma_{m j}: \quad Y_{m j}(k)=H_{m j}(k) \cdot T_{m j}(k)+\square_{m j}(k),
$$

где $H_{m j}(k)$ - матрица наблюдения, размерность и вид которой зависят от конкретной гипотезы $\Gamma_{m j} ; \square_{m j}(k)$ - вектор-столбец шумов наблюдения, представляющих собой гауссовские “белые” последовательности с нулевыми математическими ожиданиями и матрицами спектральных плотностей $N_{\square}(k)$.

Таким образом, задача синтеза алгоритма формулируется следующим образом. На основе наблюдения (5) для каждой гипотезы $\Gamma_{m j}\left(j=\overline{1, L}, \mathrm{~m}=\hat{\mathrm{m}}^{\text {(по) }}-1, \hat{\mathrm{m}}^{\text {(по) }}, \hat{\mathrm{m}}^{\text {(по) }}+1\right)$ с учетом условия (1) необходимо синтезировать алгоритм, согласно которому будет осуществляться оптимальная оценка составного вектора $\hat{\gamma}_{m j}$ (формула (2).

\section{2. Синтез алгоритма при байесовском критерии оптимальности}

Оптимальная оценка составного вектора $\hat{\gamma}_{m j}$ при байесовском критерии оптимальности заключается в определении такой его оценки, для которой минимален средний апостериорный риск $R\left[\hat{\gamma}_{m j}, Y_{m j}\right]$ (в дальнейшем для сокращения обозначений временная зависимость будет опущена), определяемый как [1,2]

$$
\begin{aligned}
& R\left[\hat{\gamma}_{m j}, Y_{m j}\right]=\sum_{g=\hat{m}^{(\text {no })}-1}^{\hat{m}^{(\text {(o) })}+1} \sum_{p=1}^{L} \int_{\left\{T_{g p}\right\}} \Pi\left[\gamma_{g p}, \hat{\gamma}_{m j}\right] \cdot W\left[\gamma_{g p} / Y_{m j}\right] d T_{g p}, \\
& m=\hat{m}^{(n o)}-1, \hat{m}^{(n o)}, \hat{m}^{(n o)}+1, \quad j=\overline{1, L},
\end{aligned}
$$

где П[$\left.\gamma_{g p}, \hat{\gamma}_{m j}\right]$ - матричная функция потерь, характеризующая потери при использовании оценки вектора $\hat{\gamma}_{m j}$, в то время, когда истинное его значение $\gamma_{g p} ; W\left[\gamma_{g p} / Y_{m j}\right]$ - апостериорная плотность вероятности оцениваемого составного вектора $\hat{\gamma}_{m j}$, которая определяется как

$$
W\left[\gamma_{g p} / Y_{m j}\right]=\frac{W\left[\gamma_{g p}\right] \cdot W\left[Y_{m j} / \gamma_{g p}\right]}{W\left[Y_{m j}\right]},
$$

где $W\left[\gamma_{g p}\right]$ и $W\left[Y_{m j}\right]$ - априорные плотности вероятности оцениваемого составного вектора $\hat{\gamma}_{m j}$ и сигнала $Y_{m j}$ соответственно; $W\left[Y_{m j} / \gamma_{g p}\right]$ - условная плотность вероятности вектора наблюдаемого сигнала $Y_{m j}$ при каждом фиксированном значении вектора $\gamma_{g p}$.

Для определения функции потерь в формуле (7) введем в рассмотрение нормированные потери, согласно которым они должны быть равны нулю при одновременном выполнении следующих условий:

$$
-157-
$$


- правильная оценка вектора $T_{m j}$;

• правильное определение количества $m$ разрешаемых по доплеровской частоте воздушных целей;

- правильное определение $j$-го набора параметров (характера) полета целей.

Если любая фазовая координата составного вектора $\gamma_{g p}$ будет оценена неверно, то потери должны быть равны единице. При этом правильная оценка вектора $T_{m j}$ будет иметь место только в том случае, когда модуль разности истинного значения вектора $T_{g p}$ и его оценки $\hat{T}_{m j}$ не будет превосходить некоторой величины $\varepsilon$, которая определяется ошибками сопровождения фазовых координат оцениваемого вектора состояния.

Исходя из этого элемент нормированной матричной функции потерь можно представить в следующем виде:

$$
\Pi\left[\gamma_{g p}, \hat{\gamma}_{m j}\right]=1-K_{g m} \cdot K_{p j}\left[1-\chi \cdot\left(\left\|\hat{T}_{m j}-T_{g p}\right\|-\varepsilon\right)\right],
$$

где

$$
\begin{aligned}
& K_{x y}=\left\{\begin{array}{l}
1, \text { при } x=y \\
0, \text { при } x \neq y
\end{array}-\right.\text { символ Кронекера; } \\
& \chi(x)=\left\{\begin{array}{l}
1, \text { при } x>0 \\
0, \text { при } x \leq 0
\end{array}-\right.\text { функция Хэвисайда; } \\
& \|\| \text { - модульное обозначение массива. }
\end{aligned}
$$

В этом случае элемент нормированной матричной функции потерь будет принимать значения «1» или «0» при следующих условиях:

$$
\Pi\left[\gamma_{g p}, \hat{\gamma}_{m j}\right]=\left\{\begin{array}{l}
1, \text { при } K_{g m}=0 ; \text { или } K_{p j}=0 ; \text { или } \chi(\cdot)=1 \\
0, \text { при } K_{g m}=K_{p j}=1 \text { и } \chi(\cdot)=0 ;
\end{array}\right.
$$

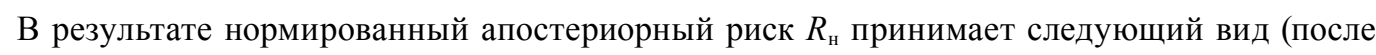
подстановки выражения (8) в формулу (6):

$$
\begin{aligned}
R_{\mathrm{H}}\left[\hat{\gamma}_{m j}, Y_{m j}\right] & =\sum_{g=\hat{m}^{\text {(о) })}-1}^{\hat{m}^{\text {(о) })}-1} \sum_{p=1}^{L} \int_{\left\{T_{g p}\right\}}\left\{1-K_{g m} K_{p j} \cdot\left[1-\chi\left(\left\|\hat{T}_{m j}-T_{g p}\right\|-\varepsilon\right)\right]\right\} \cdot W\left[\gamma_{g p}, Y_{m j}\right] d T_{g p} \\
m & =\hat{m}^{(\text {по) }}-1, \hat{m}^{(\text {по) }}, \hat{m}^{\text {(по) }}+1, \quad j=\overline{1, L}
\end{aligned}
$$

откуда следует, что

$$
\begin{aligned}
R_{\mathrm{H}}\left[\hat{\gamma}_{m j}, Y_{m j}\right]= & \sum_{g=\hat{m}^{(\mathrm{no})}-1}^{\hat{m}^{(\mathrm{no})}+1} \sum_{p=1}^{L} \int_{\left\{T_{g p}\right\}} W\left[\gamma_{g p} / Y_{m j}\right] d T_{g p}-\sum_{g=\hat{m}^{(\mathrm{no})}-1}^{\hat{m}^{(\mathrm{no})}+1} \sum_{p=1}^{L} K_{g m} K_{p j} \int_{\left\{T_{g p}\right\}} W\left[\gamma_{g p} / Y_{m j}\right] d T_{g p}+ \\
& +\sum_{g=\hat{m}^{(\mathrm{no})}-1}^{\hat{m}^{(\mathrm{no})}+1} \sum_{p=1}^{L} K_{g m} K_{p j} \int_{\left\{T_{g p}\right\}} \chi\left(\left\|\hat{T}_{m j}-T_{g p}\right\|-\varepsilon\right) \cdot W\left[\gamma_{g p} / Y_{m j}\right] d T_{g p}
\end{aligned}
$$

Третье слагаемое в формуле (10) отлично от нуля только при $\left\|\hat{T}_{m j}-T_{g p}\right\|>\varepsilon$. С учетом этого, выражение (10) преобразуется к следующему виду: 


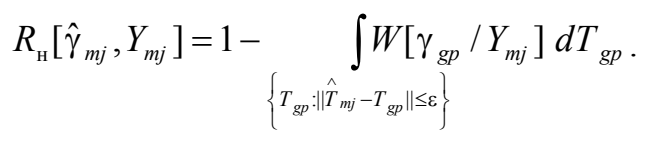

Подставляя формулу (7) в выражение (11) при функции потерь (8) получаем, что

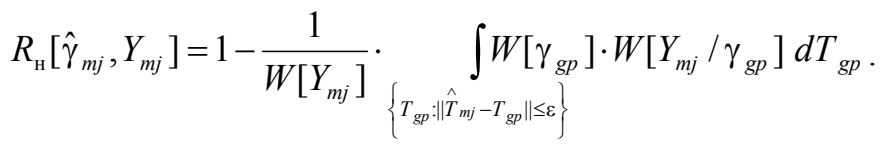

Из анализа выражения (12) следует, что минимизация апостериорного риска достигается путем максимизации величины интеграла в правой части. В результате совместная оценка $\hat{m}, \hat{j}$ и $\hat{T}$ будет определяться как

$$
\hat{\gamma}_{m j}=\arg \max _{p=1, L} \max _{g=\hat{m}^{(n o)}-1, \hat{m}^{(n o)}, \hat{m}^{(n o)}+1} \sup \left\{\int_{\left\{T_{g p}: \mid \hat{T}_{m j}-T_{g p} \| \leq \varepsilon\right\}} W\left[\gamma_{g p}\right] \cdot W\left[Y_{m j} / \gamma_{g p}\right] d T_{g p}\right\},
$$

где $\arg f(\cdot)$ - аргумент некоторой функции $f$; sup - операция определения верхней точной грани.

При условии унимодальности апостериорной плотности вероятности [3] на этапе сопровождения ГВЦ в формуле (13) можно опустить знак интеграла. В результате получим, что

$$
\hat{\gamma}_{m j}=\arg \max _{p=1, L} \max _{g=\hat{m}^{(n o)}-1, \hat{m}^{(n o)}, \hat{m}^{(n o)}+1} \sup _{\left\{T_{g p}: \mid \hat{T}_{m j}-T_{g p} \| \leq \varepsilon\right\}}\left\{W\left[\gamma_{g p}\right] \cdot W\left[Y_{m j} / \gamma_{g p}\right]\right\}
$$

откуда следует, что максимизация апостериорной плотности вероятности (минимизация нормированного апостериорного риска) должна производиться в три этапа.

На первом этапе находятся условные оценки $\hat{T}_{m j}$ при предположении о справедливости гипотезы $\Gamma_{m j}$ при каждом значении $\mathrm{m}=\hat{\mathrm{m}}^{(\text {по) }}-1, \hat{\mathrm{m}}^{(\text {по) }}, \hat{\mathrm{m}}^{\text {(по) }}+1, j=1, L$, т. е.

$$
\hat{T}_{m j}=\arg \sup _{\left\{T_{g p}\left\|\hat{T}_{m j}-T_{g p}\right\| \leq \varepsilon\right.}\left\{W\left[T_{g p}\right] \cdot W\left[Y_{m j} / T_{g p}\right]\right\} .
$$

На втором этапе определяется оценка количества воздушных целей $\hat{m}$ с учётом полученной оценки $\hat{T}_{m j}$, т. е.

$$
\hat{m}=\arg \max _{m=\hat{m}^{(n o)}-1, \hat{m}^{(n o)}, \hat{m}^{(n o)}+1}\left\{\hat{P}_{m}^{(\text {по) }} \cdot W\left[\hat{T}_{m j}\right] \cdot W\left[Y_{m j} / \hat{T}_{m j}\right]\right\} .
$$

На третьем этапе определяется оценка набора параметров полета $\hat{j}$ воздушных целей с учётом полученных оценок $\hat{T}_{m j}$ и $\hat{m}$ на первых двух этапах, как

$$
\hat{j}=\arg \max _{j=1, L}\left\{\mathrm{P}_{\hat{\mathrm{m} j}} \cdot W\left[\hat{T}_{\hat{m} j}\right] \cdot W\left[Y_{m j} / \hat{T}_{\hat{m} j}\right]\right\}
$$


Разобьём далее всю последовательность наборов параметров $j=1, L$ следующим образом: $\hat{j}=\overline{1, K}$ соответствует стационарному полету самолетов; $\hat{j}=\overline{K+1, P}$ соответствует маневрам самолетов в группе; $\hat{j}=\overline{P+1, L}$ соответствует маневрам составом группы.

На рис. 1 представлена схема синтезированного алгоритма распознавания численного состава ГВЦ и характера её полёта.

Последовательность $\hat{j}=\overline{1, K}$ может быть разбита дополнительно таким образом: $\hat{j}=\overline{1, C}$ соответствует стационарному полету самолетов в боевом порядке «пеленг» с его набором параметров; $\hat{\mathrm{j}}=\overline{\mathrm{C}+1, \mathrm{U}}$ соответствует стационарному полету самолетов в боевом порядке «клин»с его набором параметров; $\hat{\mathrm{j}}=\overline{\mathrm{U}+1, \mathrm{~K}}$ соответствует стационарному полету самолетов в боевом порядке «фронт» с его набором параметров.

В результате при таком разбиении наборов параметров полёта воздушных целей данный алгоритм позволяет параллельно с распознаванием количества целей распознать характер полёта ГВЦ по принципу «стационарный полёт-манёвр в группе-манёвр составом группы». При этом оценка вектора $\hat{T}_{\hat{m} \hat{j}}$ формируется только после принятия решения о справедливости той или иной гипотезы $\Gamma_{m j}\left(\mathrm{~m}=\hat{\mathrm{m}}^{\text {(по) }}-1, \hat{\mathrm{m}}^{\text {(по) }}, \hat{\mathrm{m}}^{\text {(по) }}+1, j=\overline{1, L}\right)$.

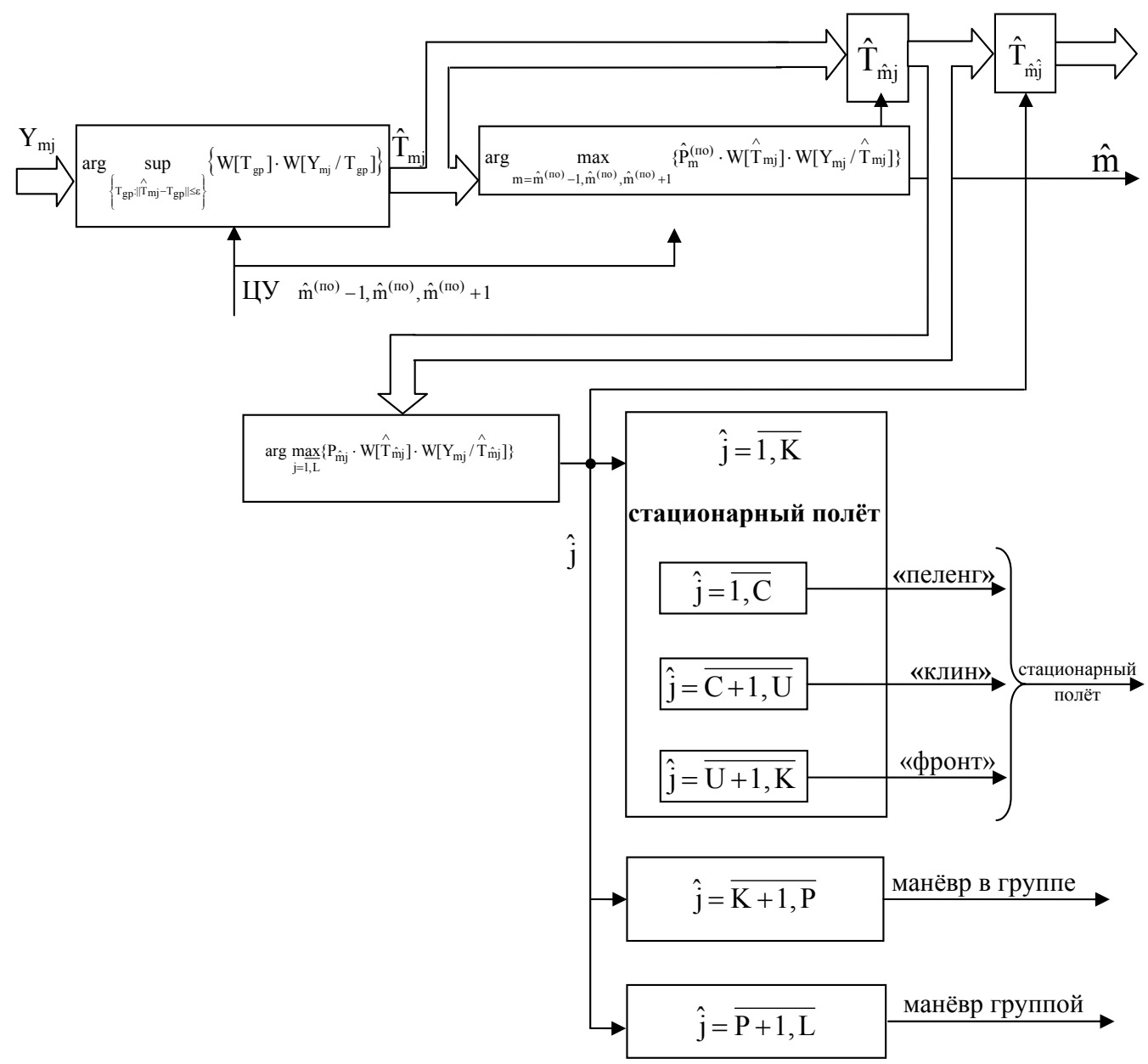

Рис. 1. Схема алгоритма распознавания количества целей и характера их полёта 


\section{2. Синтез оптимального алгоритма распознавания типового состава групповой воздушной цели}

\section{1. Постановка задачи на синтез алгоритма}

В основу распознавания типового состава ГВЦ может быть положен эффект вторичной модуляции сигналов, заключающийся в анализе взаимного частотного расположения каждой ТДЧ сигналов первичной модуляции из их совокупности и идентифицированной для данной ТДЧ (сигналов первичной модуляции) траектории доплеровской частоты сигналов вторичной модуляции из их совокупности, на основе чего определяется тип силовой установки каждого самолёта в группе из класса «самолёты с турбореактивными двигателями», а затем непосредственно и тип самого самолёта, на котором она установлена [4].

Очевидно, что вероятность распознавания типового состава ГВЦ на этапе первичной обработки сигналов невелика из-за малого времени распознавания, равного времени когерентного накопления сигнала. Данная информация является предварительной оценкой и может быть использована в виде своеобразного «целеуказания» при распознавании на этапе сопровождения ГВЦ (вторичной обработки РЛ-сигналов).

Предварительными условиями синтеза алгоритма распознавания типового состава ГВЦ являются:

РЛ-наблюдение в РЛС отражённых сигналов осуществляется в переднюю полусферу, т.е. в качестве сигналов вторичной модуляции будут приняты отражения от первых ступеней КНД;

- произведена оценка численного состава группы и характера её полёта, принято решение о том, что цели группы совершают стационарный полёт;

- количество отсчётов доплеровских частот, соответствующих первым ступеням компрессорных спектральных составляющих сигнала (индекс «К») $\mathrm{m}^{(\kappa)}$, равно оцененному количеству разрешаемых по доплеровской частоте (планерным составляющим спектра сигнала) $\hat{\mathrm{m}}$ целей в группе, т.е $\mathrm{m}^{(\mathrm{k})}=\hat{\mathrm{m}}$;

- количество типов целей в группе не превышает двух.

Пусть в каждый дискретный момент времени будет иметь место $\mathrm{m}^{(\kappa)}$ сигналов, соответ-

ствующих значениям компрессорных доплеровских частот $\mathrm{F}_{\mathrm{r}}\left(\mathrm{r}=\overline{1, \mathrm{~m}^{(\mathrm{\kappa})}}\right)$, обусловленных скоростями сближения носителя РЛС с первой ступенью КНД каждой разрешаемой по скорости (планерной составляющей спектра сигнала) ВЦ из состава группы.

Относительно каждого отсчёта доплеровской частоты, соответствующей планерной спек-

тральной составляющей $\mathrm{F}_{\mathrm{i}}(\mathrm{i}=\overline{1, \hat{\mathrm{m}}})$, сформируем разности вида

$$
\mathrm{F}_{\mathrm{i}}: \Delta \mathrm{F}_{\mathrm{ir}}=\mathrm{F}_{\mathrm{i}}-\mathrm{F}_{\mathrm{r}}, \quad \mathrm{r}=\overline{1, m^{(\kappa)}} .
$$

При $\mathrm{m}^{(\kappa)}=\hat{\mathrm{m}}$ примем гипотезу $\Gamma_{\mathrm{s}}$, которой поставим в соответствие ситуацию о том, что относительно каждого значения отсчёта доплеровской частоты $\mathrm{F}_{\mathrm{i}}(\mathrm{i}=1, \hat{\mathrm{m}})$ будут сформированы $\mathrm{m}^{(\kappa)}$ значений разностей вида (18), соответствующих s-му $(s=\overline{1, S}$ ) типу воздушной цели из состава группы.

Пусть на этапе первичной обработки сигналов установлено, что в группе существуют «q»-е и «h»-е типы целей. Тогда в интересах обеспечения высокой достоверности распознава- 
ния типового состава ГВЦ при синтезе алгоритма примем во внимание и соседние с ними типы целей, т.е. q -1, q +1, h-1, h+1. Поставим в соответствие для:

q -1 типа цели ситуацию $\mathrm{s}=1$;

q типа цели $-\mathrm{s}=2$;

$\mathrm{q}+1$ типа цели $-\mathrm{s}=3$;

$\mathrm{h}-1$ типа цели $-\mathrm{s}=4$;

$\mathrm{h}$ типа цели $-\mathrm{s}=5$;

$\mathrm{h}+1$ типа цели $-\mathrm{s}=6$.

В этом случае будет справедливо следующее условие:

$$
\mathrm{P}\left(\Gamma_{\mathrm{s}}\right)=\mathrm{P}_{\mathrm{s}}, \quad s=\overline{1, S}, \quad \sum_{s=1}^{6} P_{s}=1,
$$

где $\mathrm{P}_{\mathrm{s}}-$ вероятность справедливости гипотезы $\Gamma_{\mathrm{s}}$.

Исходя из характера решаемой задачи, оцениваемый вектор состояния относительно каждой i-й планерной доплеровской частоты $\mathrm{F}_{\mathrm{i}}(\mathrm{i}=1, \hat{\mathrm{m}})$ (при предварительно оцененном количестве целей $\hat{\mathrm{m}}$ ) будет составным и иметь следующий вид:

$$
F_{i}: \hat{\gamma}_{s}^{(i)}=\left|\hat{s}^{(i)}, \hat{T}_{s}^{(i)}(t)\right|^{T}
$$

где $s=\overline{1,6} ; \quad \mathrm{i}=\overline{1, \hat{m}} ; \hat{\gamma}_{s}^{(i)} \in G_{s}^{(i)}, \mathrm{G}_{\mathrm{s}}^{(\mathrm{i})}$ - дискретно-непрерывное множество составного векто$\wedge^{(i)}$

ра состояния; $\hat{T}_{s}(t) \in \mathrm{T}_{\mathrm{s}}^{(\mathrm{i})}(\mathrm{t}), \mathrm{T}_{\mathrm{s}}^{(\mathrm{i})}(\mathrm{t})$ - обобщенный вектор оцененных значений частных векторов состояния, определяемый как

$$
T_{(t)}^{(i)}=\left|T_{1}^{(i)}(t), \ldots, T_{s}^{(i)}(t), \ldots, T_{6}^{(i)}(t)\right|^{T},
$$

где $T_{s}^{(i)}(t)$ - оцененный частный вектор состояния, фазовыми компонентами которого для оцененного значения количества $\hat{\mathrm{m}}$ разрешаемых по доплеровской частоте целей и относительно каждой i-й планерной траектории доплеровской частоты являются оцененные значения разностей доплеровских частот

$$
T_{s}^{(i)}(t)=\Delta F_{i 1 s}(t), \ldots, \Delta F_{i r s}(t), \ldots,\left.\Delta F_{i m^{(\kappa)} 6}\right|^{T}
$$

Таким образом, относительно каждой идентифицируемой (распознаваемой) і-й планерной ТДЧ $\mathrm{F}_{\mathrm{i}}(\mathrm{t})(\mathrm{i}=1, \hat{\mathrm{m}})$ при условии $\mathrm{m}^{(\mathrm{k})}=\hat{\mathrm{m}}$ в дискретном представлении наблюдаемый сигнал $\mathrm{Y}_{\mathrm{s}}^{(\mathrm{i})}(\mathrm{k})$ на $\mathrm{k}-\mathrm{м}$ шаге при гипотезе $\Gamma_{\mathrm{s}}$ относительно варианта разноса доплеровских частот планерной и компрессорной составляющих спектра сигнала будет иметь следующий вид:

$$
m^{(\kappa)}=\hat{m}: \mathrm{F}_{\mathrm{i}}: \Delta F_{i r}: \Gamma_{s}: Y_{s}^{(i)}(k)=\Delta F_{i r s}(k)+\Upsilon_{i r s}(k)
$$

где $\Upsilon_{\mathrm{irs}}(\mathrm{k})$ - шум наблюдения, представляющий собой гауссовскую «белую» последовательность с нулевым МОЖ и спектральной плотностью $\mathrm{N}_{\mathrm{irs}}(\mathrm{k})$.

В результате задача синтеза алгоритма распознавания типового состава ГВЦ будет заключаться в том, что на основе наблюдения (23) при $\mathrm{m}^{(\kappa)}=\hat{\mathrm{m}}$, для каждой i-й распознаваемой (идентифицируемой) планерной составляющей доплеровской частоты $F_{i}(t)(i=1, \hat{m})$ и гипотезы $\Gamma_{s}(s$ 
$=\overline{1,6}$ ) относительно типа воздушной цели (разноса доплеровских частот), к которой она относится, с учетом условия (19) необходимо синтезировать алгоритм, который оптимальным образом в соответствии с заданным критерием будет производить оценку составного вектора

$\wedge^{(i)}$

$\gamma_{\mathrm{s}}$ (формула (20).

Следовательно, на основе одного и того же наблюдения необходимо:

оценить разносы траекторий доплеровских частот, обусловленных скоростями сближения РЛС с планером каждого самолёта и первыми ступенями компрессоров низкого давления каждой цели из состава группы;

оценить s-й вариант разноса доплеровских частот, характеризующий при стационарном полёте группы целей тип силовой установки конкретного самолёта, а впоследствии и его тип.

\section{1. Синтез алгоритма при байесовском критерии оптимальности}

Пусть идентификация каждой разрешаемой $\mathrm{i}$-й планерной составляющей ТДЧ $\mathrm{F}_{\mathrm{i}}(\mathrm{t})$ $(\mathrm{i}=1, \hat{\mathrm{m}})$ осуществляется независимо друг от друга. Тогда при байесовском критерии опти-

мальности оценка составного вектора $\hat{\gamma}_{s}^{(i)}$ аналогично, как и в пункте 1.2, будет заключаться в определении такой его оценки, для которой минимален средний апостериорный риск, определяемый как $[1,2]$

$$
\begin{aligned}
R\left[\hat{\gamma}_{s}^{(i)}, Y_{s}^{(i)}\right] & =\sum_{c=1}^{6} \int_{\left\{T_{c}^{(i)}\right\}} \Pi\left[\gamma_{c}^{(i)}, \hat{\gamma}_{s}^{(i)}\right] W\left[\gamma_{c}^{(i)} / Y_{s}^{(i)}\right] d T_{c}^{(i)}, \\
c, s & =\overline{1,6},
\end{aligned}
$$

где П[ $\left.\gamma_{\mathrm{c}}^{(\mathrm{i})}, \hat{\gamma}_{\mathrm{s}}^{(\mathrm{i})}\right]$ - матричная функция потерь, характеризующая потери при использовании

оценки вектора $\hat{\gamma}_{\mathrm{s}}^{(\mathrm{i})}$ в то время, когда истинное его значение $\gamma_{\mathrm{c}}^{(\mathrm{i})} ; \mathrm{W}\left[\gamma_{\mathrm{c}}^{(\mathrm{i})} / \mathrm{Y}_{\mathrm{S}}^{(\mathrm{i})}\right]-$ апостериорная плотность вероятности оцениваемого составного вектора, которая аналогично формуле (7), определяется как

$$
W\left[\gamma_{c}^{(i)} / Y_{s}^{(i)}\right]=\frac{W\left[\gamma_{c}^{(i)}\right] W\left[Y_{s}^{(i)} / \gamma_{c}^{(i)}\right]}{W\left[Y_{s}^{(i)}\right]},
$$

где $\mathrm{W}\left[\gamma_{c}^{(i)}\right]$ и $\mathrm{W}\left[Y_{s}^{(i)}\right]$ - априорные плотности вероятности оцениваемого составного вектора и наблюдаемого сигнала соответственно; $\mathrm{W}\left[Y_{s}^{(i)} / \gamma_{c}^{(i)}\right]$ - условная плотность вероятности вектора наблюдаемого сигнала $Y_{s}^{(i)}$ при каждом фиксированном значении вектора $\gamma_{c}^{(i)}$.

Выбор функции потерь в (24) определим, как и в п. 1.2. Потери должны быть равны нулю при одновременном выполнении следующих условий:

- при правильной оценке вектора $T_{s}^{(i)}$;

- при правильном определении s-го варианта разноса доплеровских частот планерной и компрессорной составляющих спектра сигнала, соответствующего s-му типу воздушной цели, к которой относится і-я планерная траектория доплеровской частоты.

$$
-163-
$$


Если любая фазовая координата составного вектора $\gamma_{c}^{(i)}$ будет оценена неверно, то потери должны быть равны единице. При этом правильная оценка вектора $T_{c}^{(i)}$ будет иметь место только в том случае, когда модуль разности истинного значения вектора $T_{c}^{(i)}$ и его оценки $T_{c}^{(i)}$ не будет превосходить некоторой величины $\varepsilon^{*}$, которая определяется ошибками оценки фазовых компонент оцениваемого вектора состояния.

В результате элемент нормированной матричной функции потерь можно представить в следующем виде:

$$
\Pi\left[\gamma_{c}^{(i)}, \hat{\gamma}_{s}^{(i)}\right]=1-K_{c s}\left[1-\chi\left(\left\|\hat{T}_{s}^{(i)}-T_{c}^{(i)}\right\|-\varepsilon^{*}\right)\right]
$$

где

$$
\begin{aligned}
& K_{c s}=\left\{\begin{array}{l}
1, \text { при } c=s, \\
0, \text { при } c \neq s,
\end{array}-\right.\text { символ Кронекера; } \\
& \chi(x)=\left\{\begin{array}{l}
1, \text { при } x>0, \\
0, \text { при } x \leq 0,
\end{array},-\right. \text { функция Хевисайда, }
\end{aligned}
$$

откуда следует, что он будет принимать значения “1” или “0” при следующих условиях:

$$
\Pi\left[\gamma_{c}^{(i)}, \hat{\gamma}_{s}^{(i)}\right]=\left\{\begin{array}{l}
1, \text { при } K_{c s}=0 ; \text { или } \chi(\cdot)=1 ; \\
0, \text { при } K_{c s}=1 \text { и } \chi(\cdot)=0 .
\end{array}\right.
$$

В результате нормированный апостериорный риск $\mathrm{R}_{\text {н }}$ принимает следующий вид:

$$
\begin{aligned}
& \left.R_{\mathrm{H}} \hat{[}_{s}^{(i)}, Y_{s}^{(i)}\right]=\sum_{c=1}^{6} \int_{\left\{T_{c}^{(i)}\right\}}\left\{1-K_{c s}\left[1-\chi\left(\left\|\hat{T}_{s}^{(i)}-T_{c}^{(i)}\right\|-\varepsilon^{*}\right)\right]\right\} W\left[\gamma_{c}^{(i)}, Y_{s}^{(i)}\right] d T_{c}^{(i)} \\
& \quad c, s=\overline{1,6},
\end{aligned}
$$

откуда вытекает, что

$$
\begin{gathered}
R_{\mathrm{H}}\left[\hat{\gamma}_{s}^{(i)}, Y_{s}^{(i)}\right]=\sum_{c=1}^{6} \int_{\left\{T_{c}^{(i)}\right\}} W\left[\gamma_{c}^{(i)} / Y_{s}^{(i)}\right] d T_{c}^{(i)}-\sum_{c=1}^{6} K_{c s} \int_{\left\{T_{c}^{(i)}\right\}} W\left[\gamma_{c}^{(i)} / Y_{s}^{(i)}\right] d T_{c}^{(i)}+ \\
+\sum_{c=1}^{6} K_{c s} \int_{\left\{T_{c}^{(i)}\right\}} \chi\left(\left\|\hat{T}_{s}^{(i)}-T_{c}^{(i)}\right\|-\varepsilon^{*}\right) W\left[\gamma_{c}^{(i)} / Y_{s}^{(i)}\right] d T_{c}^{(i)} .
\end{gathered}
$$

При условии, что третье слагаемое в выражении (28) отлично от нуля при $\left\|\hat{\mathrm{T}}_{\mathrm{s}}-\mathrm{T}_{\mathrm{c}}^{(\mathrm{i})}\right\|>\varepsilon^{*}$, оно преобразуется к следующему виду:

$$
\begin{gathered}
R_{\mathrm{H}}\left[\hat{\gamma}_{s}^{(i)}, Y_{s}^{(i)}\right]=1-\int W\left[\gamma_{c}^{(i)} / Y_{s}^{(i)}\right] d T_{c}^{(i)}, \\
\left\{T_{c}^{(i)}:\left\|\hat{T}_{s}^{(i)}-T_{c}^{(i)}\right\| \leq \varepsilon^{*}\right\}
\end{gathered}
$$

откуда с учётом формул (25) и (26) получаем выражение для нормированного апостериорного риска:

$$
\begin{gathered}
R_{\mathrm{H}}\left[\hat{\gamma}_{s}^{(i)}, Y_{s}\right]=1-\frac{1}{W\left[Y_{s}\right]} \underset{\left\{T_{c}^{(i)}: \| \hat{T}_{s}^{(i)}-T_{c}^{(i)} \leq \varepsilon^{*}\right\}}{ }\left[\begin{array}{l}
(i) \\
-164-
\end{array}\right. \\
\left.-1 Y_{s} / \gamma_{c}^{(i)}\right] d T_{c}^{(i)} .
\end{gathered}
$$


путем максимизации величины интеграла в правой части. В результате совместная оценка S и $\wedge^{(i)}$

$\hat{\mathrm{T}}_{\mathrm{s}}$ будет определяться выражением

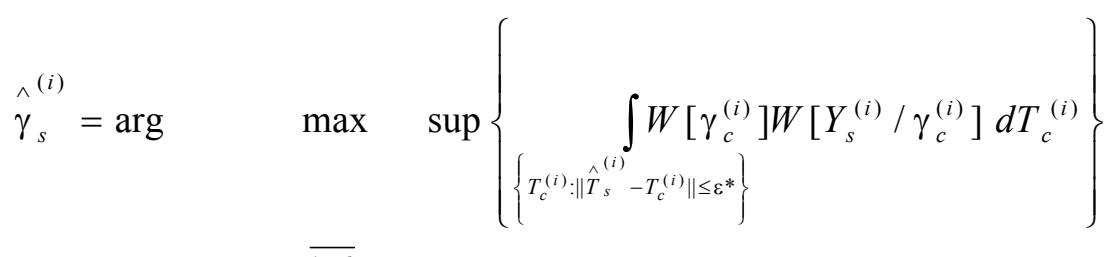

$$
\begin{aligned}
& s=\overline{1,6}
\end{aligned}
$$

и при унимодальности апостериорной плотности вероятности [3] преобразуется к следующему виду:

$$
\begin{array}{r}
\hat{\gamma}_{s}^{(i)}=\arg \quad \max \left\{W\left[\gamma_{c}^{(i)}\right] W\left[Y_{s}^{(i)} / \gamma_{c}^{(i)}\right\}\right. \\
s=\overline{1,6} \quad\left\{T_{c}^{(i)}:\left\|\hat{T}_{s}^{(i)}-T_{c}^{(i)}\right\| \leq \varepsilon^{*}\right\}
\end{array}
$$

Из формулы (32) можно сделать вывод о том, что максимизация апостериорной плотности вероятности (минимизация нормированного апостериорного риска) должна производиться в два этапа.

На первом этапе находят условные оценки $\hat{T}_{s}$ при предположении о справедливости гипотезы $\Gamma_{\mathrm{s}}$ при каждом значении $s=\overline{1,6}$, т. е.

$$
\begin{aligned}
\hat{T}_{s}^{(i)}=\arg & \sup \left\{W\left[T_{c}^{(i)}\right] W\left[Y_{s}^{(i)} / T_{c}^{(i)}\right\}\right. \\
& \left\{T_{c}^{(i)}:\left\|\hat{T}_{s}^{(i)}-T_{c}^{(i)}\right\| \leq \varepsilon^{*}\right\}
\end{aligned}
$$

На втором этапе определяется оценка варианта разноса доплеровских частот $\hat{\mathrm{s}}$ (соответствует s-му типу цели) с учётом полученной оценки $\hat{T}_{s}^{(i)}$, как

$$
\hat{s}=\arg \max \left\{P_{s} W\left[\hat{T}_{s}^{(i)}\right] W\left[Y_{s}^{(i)} / \hat{T}_{s}^{(i)}\right]\right\} .
$$

На рис. 2 представлена схема синтезированного при байесовском подходе алгоритма распознавания типового состава ГВЦ.

Таким образом, синтезированный алгоритм позволяет параллельно с распознаванием количества целей в группе, характером их полёта и в случае их стационарного полёта распознать на этапе вторичной обработки сигналов типовой состав групповой воздушной цели. 


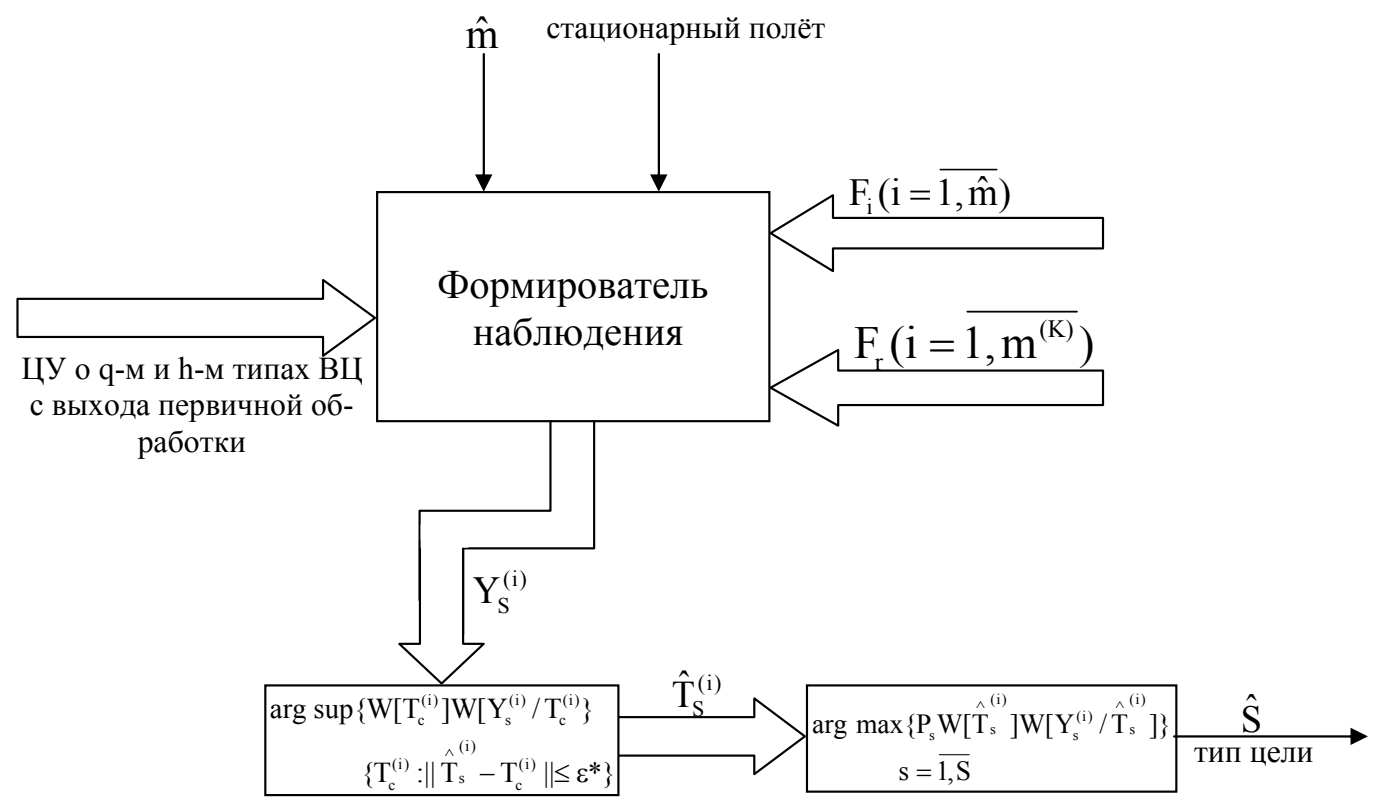

Рис. 2. Схема алгоритма распознавания типового состава ГВЦ

\section{Заключение}

Синтезированы при общем байесовском критерии оптимальности алгоритмы распознавания численного и типового состава ГВЦ, характера её полёта. На рис. 3 представлена схема системы, объединяющая синтезированные алгоритмы.

На вход системы алгоритмов распознавания поступают: отчеты доплеровских частот с выхода блока БПФ; информация с выхода первичной обработки сигналов о количестве целей в группе и их типе.

Изначально распознаётся численный состав ГВЦ. Данная информация применяется для распознавания характера полёта ГВЦ, а также её типового состава, где используется информация о том, что цели совершают стационарный полёт.

В результате на выходе системы вторичной обработки сигналов при распознавании ГВЦ формируется информация о её численном и типовом составах, а также характере полёта самолётов в группе в таких основных формах боевых порядков, как «пеленг», «клин», «фронт». 


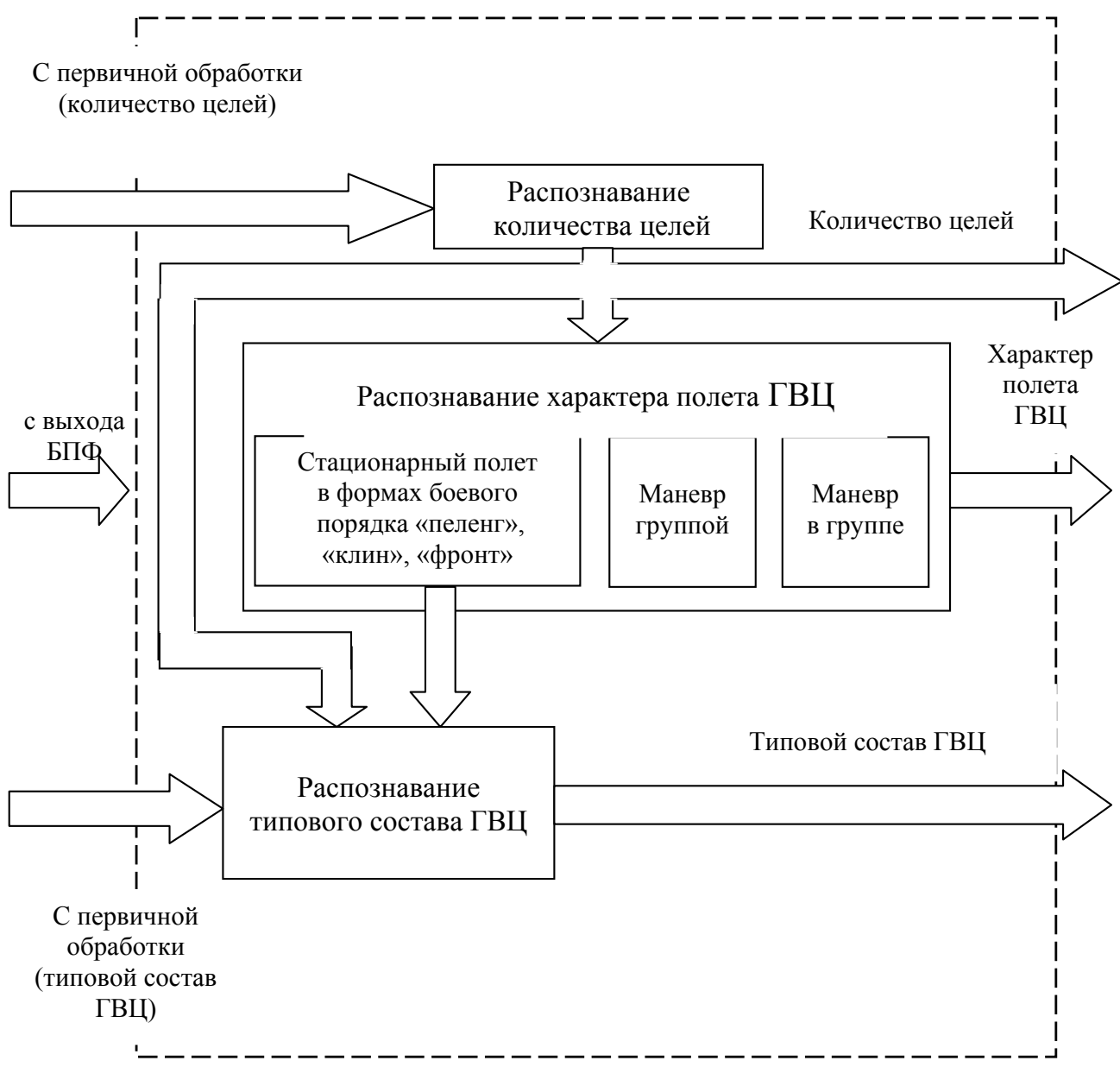

Рис. 3. Система синтезированных алгоритмов

\section{Список литературы}

[1] Трифонов А.П., Шинаков Ю.С. Совместное различение сигналов и оценка их параметров на фоне помех. М.: Радио и связь, 1986 [Trifonov A.P., Shinakov J.S. Joint distinction of signals and estimation of their parameters on the background noise. Moscow, Radio and Communications, 1986 (in Russian)].

[2] Садовников А.П. Оптимальное совместное обнаружение-разрешение-оценивание неизвестного числа сигналов и их параметров при пространственно-временных траекторных наблюдениях. Радиотехника, 1990, 12 [Sadovnikov A.P. The optimal joint detection, resolution-evaluation of an unknown number of signals and their parameters in the space-time trajectory observations, Radio engineering, 1990, 12 (in Russian)].

[3] Ярлыков М.С. Статистическая теория радионавигации. М.: Радио и связь, 1985. [Jarlikov M.S. Statistical theory radionavigatsii. Moscow, Radio and Communications, 1985 (in Russian)].

[4] Патент 2456633. Российская Федерация, МПК G01S 13/52 (2006.01). Способ сопровождения групповой воздушной цели из класса «самолеты с турбореактивными двигателями». А.Г. Ситников, А.В. Богданов, О.В. Васильев, А.К. Ибрагим, А.А. Филонов, Ю.А. Круталевич; заяви- 
тель и патентообладатель Федеральное государственное научное учреждение "Государственный научно-технологический центр “Наука” (ФГНУ “ГНТЦ “Наука”) (RU). № 2011117802/07; заявл. 03.05.2011; опубл. 20.07.2012. Бюл. № 20. 14 с.: ил. [Patent 2456633 Russian Federation, IPC G01S 13/52 (2006.01). A method of tracking a group of air target class "aircraft with turbojet engines". A.G. Sitnikov, A.V. Bogdanov, O.V. Vasiliev, A.K. Ibrahim, A.A. Filonov Yu.A. Krutalevich; the applicant and the patentee Federal State Scientific Institution "State Science and Technology Center" Science "(Federal State" SSTC "Science")( in Russian)]. 